\title{
Relationship between solar wind corotating interaction regions and the phasing and intensity of Saturn kilometric radiation bursts
}

\author{
S. V. Badman ${ }^{1}$, S. W. H. Cowley ${ }^{1}$, L. Lamy ${ }^{2}$, B. Cecconi ${ }^{2}$, and P. Zarka ${ }^{2}$ \\ ${ }^{1}$ Department of Physics \& Astronomy, University of Leicester, Leicester LE1 7RH, UK \\ ${ }^{2}$ LESIA, Observatoire de Paris, Bâtiment 16, 5 Place Jules Janssen, 92195 Meudon, France
}

Received: 24 April 2008 - Revised: 12 September 2008 - Accepted: 21 October 2008 - Published: 19 November 2008

\begin{abstract}
Voyager spacecraft measurements of Saturn kilometric radiation (SKR) identified two features of these radio emissions: that they pulse at a period close to the planetary rotation period, and that the emitted intensity is correlated with the solar wind dynamic pressure (Desch and Kaiser, 1981; Desch, 1982; Desch and Rucker, 1983). In this study the inter-relation between the intensity and the pulsing of the SKR is analysed using Cassini spacecraft measurements of the interplanetary medium and SKR over the interval encompassing Cassini's approach to Saturn, and the first extended orbit. Cassini Plasma Spectrometer ion data were only available for a subset of the dates of interest, so the interplanetary conditions were studied primarily using the nearcontinuously available magnetic field data, augmented by the ion moment data when available. Intense SKR bursts were identified when solar wind compressions arrived at Saturn. The intensity of subsequent emissions detected by Cassini during the compression intervals was variable, sometimes remaining intense for several planetary rotations, sometimes dimming and rarely disappearing. The timings of the initial intense SKR peaks were sometimes independent of the longterm pulsing behaviour identified in the SKR data. Overall, however, the pulsing of the SKR peaks during the disturbed intervals was not significantly altered relative to that during non-compression intervals.
\end{abstract}

Keywords. Magnetospheric physics (Planetary magnetospheres; Solar wind-magnetosphere interactions) - Solar physics, astrophysics, and astronomy (Radio emissions)

Correspondence to: S. V. Badman

(svb4@ion.le.ac.uk)

\section{Introduction}

In 1980 the Voyager spacecraft made the first detections of kilometre wavelength radiation from Saturn (Kaiser et al., 1980). These Saturn kilometric radiation (SKR) emissions cover a broad frequency range from a few $\mathrm{kHz}$ to $\sim 1200 \mathrm{kHz}$, with a spectral peak at $\sim 100-400 \mathrm{kHz}$. They are believed to be generated by the cyclotron maser instability (CMI) mechanism, first proposed to explain terrestrial auroral radio emissions by Wu and Lee (1979), whereby the radio wave is excited by energy lost from electrons on auroral field lines. The Voyager measurements revealed two features of the SKR emissions. First, the emission was modulated at a period close to the rotation period of the planet, and second, the SKR emitted power was positively correlated with the solar wind dynamic pressure (Desch and Kaiser, 1981; Desch, 1982; Desch and Rucker, 1983). Regarding the first of these features, measurements by Ulysses and Cassini have established that the SKR period is not constant but has changed significantly from the Voyager value of $10 \mathrm{~h} 39 \mathrm{~min} 24 \mathrm{~s} \pm 7 \mathrm{~s}$ to $10 \mathrm{~h} 45 \mathrm{~min} 45 \mathrm{~s} \pm 36 \mathrm{~s}$ during Cassini approach, and is subject to variations over shorter timescales (e.g. Galopeau and Lecacheux, 2000; Gurnett et al., 2005). In addition, Zarka et al. (2007) concluded from analysis of an extended interval of Cassini data that the solar wind velocity is correlated with short-term (i.e. 20-30 day) fluctuations in the period of the SKR emissions. Regarding the emitted SKR power, recent case studies using Cassini data have suggested that a solar wind corotating interaction region (CIR) compression of Saturn's magnetosphere can lead to intensification and then drop-out (i.e. non-detection) of the modulated SKR emissions (e.g. Bunce et al., 2005; Jackman et al., 2005; Kurth et al., 2005a; Mitchell et al., 2005). None of these studies has yet revealed how the intensity and pulsing of the SKR emissions are interrelated under solar wind compression conditions. Our study thus concentrates on this relationship, by

Published by Copernicus Publications on behalf of the European Geosciences Union. 
looking for intensifications of the SKR power following arrival of a solar wind compression, any subsequent "drop-out" of the emission detected by Cassini, the phasing of the intensified or reduced emission peaks during the compression event, and the relative phasing and intensity of the emission peaks before and after the compression.

Cassini data from the magnetometer (MAG) (Dougherty et al., 2004), Cassini Plasma Spectrometer (CAPS) (Young et al., 2004) and Radio and Plasma Wave Science (RPWS) investigation (Gurnett et al., 2004) are presented to examine the interaction of compressions in the solar wind with the intensity and pulsing of the SKR emission. Data obtained during Cassini's approach to Saturn (October 2003-June 2004) and its first extended orbit following Saturn orbit insertion (SOI) (July-October 2004) were analysed. These were intervals when Cassini was measuring both the interplanetary conditions upstream of Saturn and SKR emissions from Saturn. Since CAPS solar wind ion data were only available for a subset of the dates of interest, the interplanetary conditions were studied primarily using the near-continuously available magnetic field data, augmented by the ion moment data when available. In the following sections details of the format of the Cassini data employed in this study, and the modelled drifting period of SKR bursts are given. Section 4 describes a selection of the CIR compression events in detail, then summarises the features of all events included in the study. Finally, some general conclusions are drawn about the effects of solar wind compressions on the power and modulation of the detected SKR.

\section{Cassini measurements of SKR emissions and inter- planetary magnetic field strength}

The Cassini data used in this study are from late 2003 (day 344) until Cassini encountered Saturn's magnetosphere on day 179 of 2004, and then days 195-298 of 2004 when Cassini had exited Saturn's magnetosphere back into the solar wind. Ideally the solar wind dynamic pressure would be presented to compare with the Voyager results but this data is only available for a modest subset of the events studied here. However, due to the frozen-in nature of the solar wind flow, the interplanetary magnetic field (IMF) magnitude measured by Cassini MAG can be used as a proxy for the solar wind dynamic pressure. In general, a compression region in the solar wind will be observed as an increase in IMF magnitude, bounded by forward and reverse shocks (Smith and Wolfe, 1976; Gosling and Pizzo, 1999). As heliospheric current sheet (HCS) crossings usually occur within CIR compression regions in the solar wind, a reversal in the sense of the $B_{T}$ interplanetary field component (RTN coordinates) can further be used to identify a CIR compression event (Gosling and Pizzo, 1999). (The RTN coordinate system is a righthanded spherical polar system referenced to the Sun's spin axis, with $B_{R}$ directed radially outward from the Sun, $B_{T}$ az- imuthal in the direction of solar rotation, and $B_{N}$ normal to the other two components, that is, positive northwards from the equatorial plane.) When solar wind dynamic pressure data were available from CAPS they also have been analysed and found to show an excellent correspondence with the signatures of compression regions identified in the IMF measurements. One example demonstrating this correspondence during the post-SOI interval will be presented in Sect. 4 below. Thirteen CIR compression events with good MAG and RPWS data coverage (including five with simultaneous solar wind density and velocity data from CAPS) were identified during the intervals of interest and will be described below.

The SKR data are presented in two forms in this study to help identify the features within it. The first is a colourcoded electric field spectrogram from the Cassini RPWS instrument, which shows the power in $\mathrm{W} \mathrm{m}^{-2} \mathrm{~Hz}^{-1}$ normalized to a distance of $1 \mathrm{AU}$ in each frequency channel in the range $3.5 \mathrm{kHz}$ to $1.5 \mathrm{MHz}$, at $3 \mathrm{~min}$ resolution. The SKR emitted power integrated over the SKR peak frequency band of $100-400 \mathrm{kHz}$ is also shown. Both these data sets have been "cleaned" to remove non-SKR emissions e.g. solar radio emissions and spacecraft noise, and the occurrence times corrected for the planet-spacecraft light travel time (Lamy et al., 2008). The SKR emitted power data are averaged over a time interval of approximately one twentieth of the radio rotation period to enable good resolution of the emission bursts. Due to the drifting period of the SKR we use two nominal SKR periods derived from Cassini data to determine the cadence of the averaged emitted power data, one pre-SOI and one post-SOI. The pre-SOI data uses a characteristic period of $10.7625 \mathrm{~h}$ (Gurnett et al., 2005), such that the data are $0.538125 \mathrm{~h}$ averages. The post-SOI data uses a characteristic period of $10.7811 \mathrm{~h}$ (Kurth et al., 2005b) i.e. the power data are $0.539056 \mathrm{~h}$ averages. We emphasise that these values determine only the cadence of the plotted data, the difference between them not being critical to the results presented. When considering the timing of the SKR pulses the full varying SKR period determined for this interval by Kurth et al. (2007) will be employed, as discussed in Sect. 3 below. In late 2003-2004 Saturn's Southern Hemisphere was tilted significantly towards the Sun. Most of the SKR emissions detected by Cassini, which was approaching close to the ecliptic plane, therefore originated in the Southern Hemisphere i.e. were left-hand ( $\mathrm{LH})$ circularly-polarized. The RH emissions at Cassini were very weak in comparison therefore the $\mathrm{LH}$ power was used as an approximation for the total SKR power detected by Cassini.

\section{Drifting period of SKR emissions}

One of the purposes of this study is to determine whether solar wind compressions significantly disrupt or shift the pulsing of the SKR peaks, therefore the expected times of the pulses based on their long-term behaviour must be known for 
comparison with those observed. Kurth et al. (2007) derived an expression for the variation of the SKR phase relative to a fixed period $\left(T_{0}=0.4497 \mathrm{~d}\right)$ by fitting a third order polynomial to Cassini measurements of the timing of the SKR peaks over the interval from 1 January 2004 to 28 August 2006. This phase drift as a function of time $t$ in days since 1 January 2004 is given by

$\Delta \Phi_{\mathrm{SKR}}(t)=C_{1}+C_{2} t+C_{3} t^{2}+C_{4} t^{3}$,

where $C_{1}=87.77( \pm 10.1)^{\circ}$,

$C_{2}=-2.527\left( \pm 9.05 \times 10^{-2}\right)^{\circ} \mathrm{d}^{-1}$,

$C_{3}=3.041 \times 10^{-3}\left( \pm 2.17 \times 10^{-4}\right)^{\circ} \mathrm{d}^{-2}$ and

$C_{4}=-7.913 \times 10^{-7}\left( \pm 1.47 \times 10^{-7}\right)^{\circ} \mathrm{d}^{-3}$. This phase drift

$\Delta \Phi_{\mathrm{SKR}}$ is subtracted from that of the fixed period to give the phase of the SKR at any time:

$\Phi_{\mathrm{SKR}}(t)=\frac{360}{T_{0}} \times t-\Delta \Phi_{\mathrm{SKR}}(t)$.

The drifting SKR period $T_{\mathrm{SKR}}$ is then given by:

$T_{\mathrm{SKR}}(t)=\frac{360}{d \Phi_{\mathrm{SKR}} / d t}=\frac{360}{\left(\frac{360}{T_{0}}-\left(C_{2}+2 C_{3} t+3 C_{4} t^{2}\right)\right)}$.

These functions are defined such that when $\Phi_{\mathrm{SKR}}(t)=360 n$, where $n$ is an integer, there should be a peak in the SKR emission. To locate the times of the expected SKR peaks we therefore set Eq. (2) equal to $360 n$, incorporating Eq. (1), and solve for successive $n$ to find a set of times $t$. The errors in the constants $C_{1}, C_{2}$, etc. given above define the accuracy of the Kurth et al. (2007) polynomial fit to the measured SKR peaks, which corresponds to a maximum "error" in the SKR period of $\pm 25 \mathrm{~s}$ over the intervals studied here. However, the spread in the measured timings of the SKR peaks is actually significantly larger than this, as shown by Kurth et al. (2007) (see their Fig. 2), and is partially attributed to the broadness of the peaks of the SKR emission. Zarka et al. (2007) also showed there are in fact short-term variations ( $\pm 6 \mathrm{~min})$ in the SKR period during the intervals of interest, associated with the solar wind speed. In this study we therefore use the "expected" times of the peaks in SKR emission (" $t$ ") as a guide to highlight any major systematic changes in the modulation of the SKR associated with solar wind compression events, on much larger scales than those identified by Zarka et al. (2007). In the next section examples of solar wind CIR events and the corresponding SKR detections are presented and discussed.

\section{Examples of solar wind compression events and the corresponding SKR detections}

The sections below detail three solar wind compression events from 2004, each displaying different characteristics, and the varying responses in the SKR emissions detected by Cassini. In chronological order (as they will be discussed below) these events are: 1) a HCS crossing identified by a reversal in IMF $\mathrm{B}_{T}$ without a large change in field magnitude; 2) a minor CIR compression region; 3) a major CIR compression bounded by both forward and reverse shocks. In each case the data from a few days either side of the compression are shown to illustrate the nature of the preceding and subsequent SKR bursts for comparison. CAPS ion data are only available for event 3 . A summary of the features of all thirteen events is then discussed.

\subsection{Days 49-55 of 2004}

The Cassini data acquired over days 49-55 (18-24 February) of 2004, when Cassini was at a radial distance from Saturn of more than $1000 R_{S}$, are shown in Fig. 1. The top panel shows the emitted SKR power corrected for radial distance in Watts per steradian, over the frequency range of $100-300 \mathrm{kHz}$. The crosses toward the top of the panel mark the timings of the expected SKR peaks according to the Kurth et al. (2007) algorithm described above. The middle panel of Fig. 1 is an electric field spectrogram, where the relative powers are colour-coded according to the colour bar on the right hand side of the figure, and plotted as a function of frequency and time. The crosses in the upper part of this panel show the expected timings of the SKR peaks as in the upper panel. The bottom panel shows the IMF magnitude $|\boldsymbol{B}|$ in nT, colourcoded according to the sense of IMF $B_{T}$, where blue represents $B_{T}<0$ and red represents $B_{T}>0$, as indicated at the right hand side of the panel. The time axis is labelled at intervals of days, with Cassini's radial distance from Saturn also labelled in units of Saturn radii (here $1 R_{S}=60268 \mathrm{~km}$ ). The time taken for the solar wind to propagate from the spacecraft to the planet, assuming purely radial motion and using a nominal solar wind speed of $500 \mathrm{~km} \mathrm{~s}^{-1}$, is given at the top of the figure. The actual propagation delay is very uncertain (to many hours) due to possible non-radial propagation of the solar wind and variations in the flow speed (Crary et al., 2005). In the example shown in Fig. 1 the radial propagation delay is nominally $\sim 15 \mathrm{~h}$, varying by $\pm 0.5 \mathrm{~h}$ over the interval due to Cassini's motion towards Saturn, reducing to $\sim 11 \mathrm{~h}$ for propagation of phase fronts aligned along the Parker spiral direction. The IMF data plotted in the bottom panel is lagged by the radial propagation delay of $\sim 15 \mathrm{~h}$ to indicate how they may correspond to the detected SKR emissions. The vertical dashed lines identify selected SKR features and the corresponding lagged IMF data that will be discussed below.

The event shown in Fig. 1 began with "quiet" conditions on days $49-50$ of 2004 . Looking first at the lagged IMF data in the bottom panel, the field magnitude at this time was relatively constant at $\sim 0.8 \mathrm{nT}$, before reducing at $\sim 02: 00$ UT on day 51 , then increasing to $\sim 1.1 \mathrm{nT} \sim 10 \mathrm{~h}$ later. This change in field magnitude was coincident with a reversal of the $B_{T}$ component (positive to negative) indicating a HCS crossing. For the next three days the field remained 


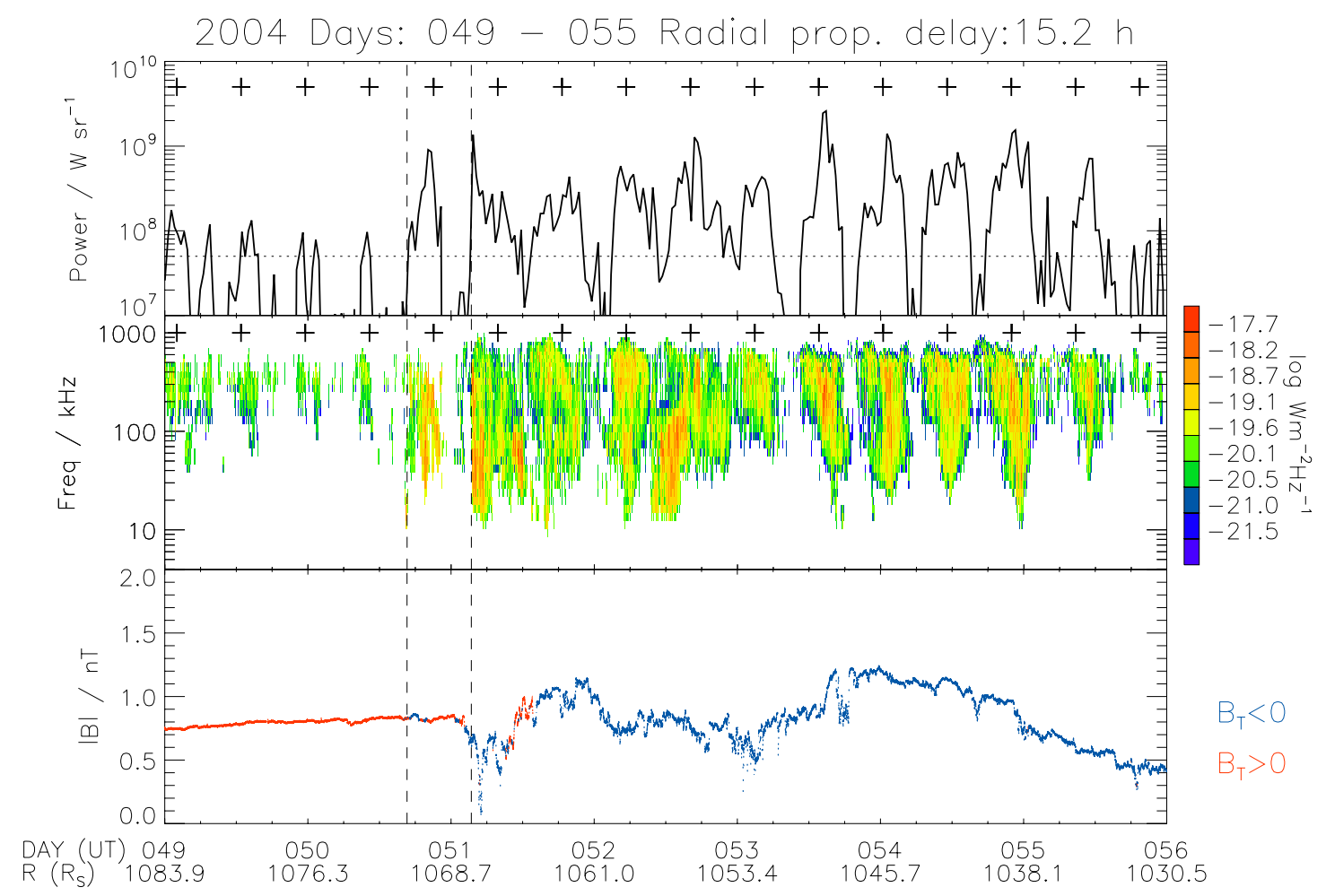

Fig. 1. SKR and IMF data from days 49-55 of 2004. The top panel shows $0.538125 \mathrm{~h}$ averages of the emitted power of the SKR integrated over $100-300 \mathrm{kHz}$. The crosses toward the top of the panel mark the expected times of the SKR peaks given by Kurth et al. (2007), as described in the text. The middle panel shows the SKR electric field power as a function of frequency and time, colour-coded according to the colour bar at the right hand side of the figure. The crosses on this panel represent the expected peaks of SKR emission, as in the top panel. The bottom panel is a plot of the interplanetary magnetic field strength in nT, colour coded by the sense of the RTN $B_{T}$ component as indicated at the right hand side of the panel. The time axis of these plots is marked in units of days, with sub-divisions every 6 h. Also marked is the spacecraft range from Saturn in units of Saturn radii (where $1 R_{S}=60268 \mathrm{~km}$ ). At the top of the plot, an estimate of the solar wind propagation delay is marked, estimated assuming purely radial propagation and a constant solar wind speed of $500 \mathrm{~km} \mathrm{~s}^{-1}$. The vertical dashed lines mark features described in the text.

disturbed, fluctuating on short timescales between $\sim 0.5 \mathrm{nT}$ and $\sim 1.2 \mathrm{nT}$. At $\sim 23: 00 \mathrm{UT}$ on day 54 the field magnitude began a steady decline, reaching $\sim 0.4 \mathrm{nT}$ at the end of day 55. We identify the HCS crossing and disturbed field on day 51 as the arrival of a minor compression region at Cassini, and now examine its effects on the SKR emissions detected.

The data in the upper two panels of Fig. 1 show that the SKR emissions have variable structure. For the purposes of describing the data in this section we define a burst of SKR as an interval of emission above $5 \times 10^{7} \mathrm{~W} \mathrm{sr}^{-1}$ (i.e. approximately twice the median value of $2.7 \times 10^{7} \mathrm{~W} \mathrm{sr}^{-1}$ determined by Lamy et al., 2008), marked in the top panel of Fig. 1 by the horizontal dotted line, and the burst peak as the local maximum in emission. Using these definitions we identify seven SKR bursts during days 49-50. The peaks occurred close to the "expected" times, indicated by the crosses in the upper two panels, except for the second burst on day 49 , which occurred approximately half way between two expected peaks, and the brief fifth burst at the start of day 50. The power of each of these peaks was $\sim 10^{8} \mathrm{~W} \mathrm{sr}^{-1}$ until the last $(\sim 21: 00$ UT on day 50$)$ which reached a maximum power of $\sim 10^{9} \mathrm{~W} \mathrm{sr}^{-1}$, i.e. an order of magnitude larger. The start of this burst is marked by the first vertical dashed line. This was followed by another SKR burst, indicated by the second vertical dashed line, which peaked just $\sim 8 \mathrm{~h}$ later, i.e. before the next expected time of a peak marked by the cross. The timing of this peak was $\sim 16 \mathrm{~h}$ (not accounting for the propagation delay) after the IMF magnitude decreased, indicating the start of the field disturbance. Because this SKR burst began and peaked significantly before the expected time of the next regular pulsed emission we now it an "extra" burst, but note that it continued at lower powers for $\sim 9 \mathrm{~h}$, encompassing the next expected peak time. This burst could mark the arrival of the disturbed interplanetary conditions at Saturn. However, the increased power of the previous burst suggests that perhaps the field disturbance arrived at this earlier time. It is not possible to confirm which of these is the initial response of the SKR however, because there was no sharp shock front in the field data 


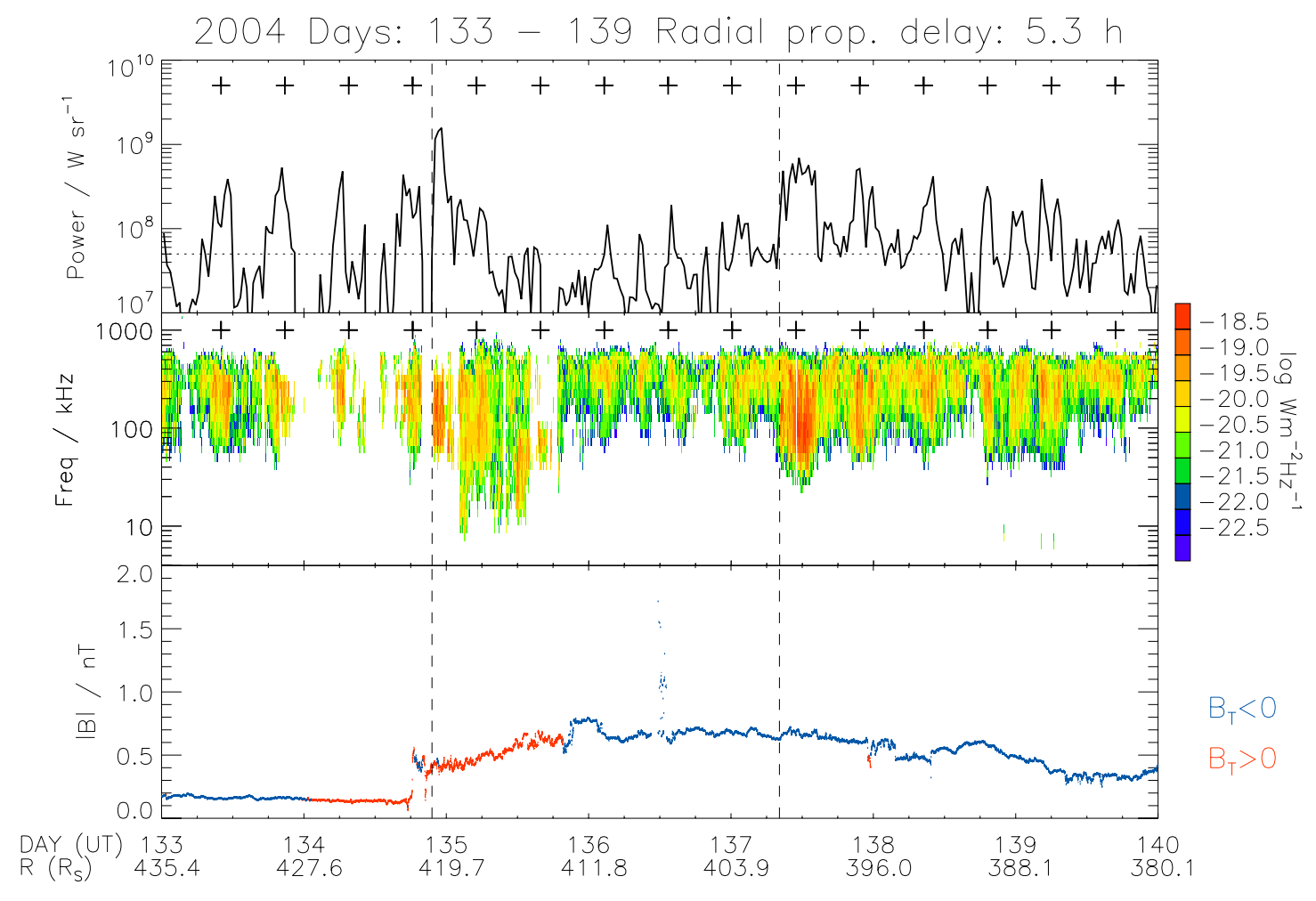

Fig. 2. SKR and IMF data from days 133-139 of 2004, in the same format as Fig. 1.

to identify the start of the compression, and there are significant uncertainties in the estimated propagation delay, as described above. Both of these bursts extend to lower frequencies than the preceding bursts $(\sim 10 \mathrm{kHz}$ compared to $50-100 \mathrm{kHz}$ ). This is a feature of SKR emission previously noted during a compression interval by Kurth et al. (2005a). For the purposes of our analysis the second of these bursts was selected as the initial post-compression burst due to its correspondence with the IMF $B_{T}$ reversal, and assuming an accurate propagation delay. Over the following four days there were several long-lived ( $>6 \mathrm{~h}$ ), intense SKR bursts, all at powers of $\sim 10^{9} \mathrm{~W} \mathrm{sr}^{-1}$. The final SKR burst shown on day 55, when the field strength was declining, had a lower power of $\sim 10^{8} \mathrm{~W} \mathrm{sr}^{-1}$, similar to that of the bursts before the field disturbance. These results are also listed in Table 1 (see event number 6), to be discussed in detail below. When constructing Table 1 only bursts which peaked closest to the expected times are included to enable analysis of any changes in the pulsing of the bursts. All other bursts were treated as "extra" emissions as explained above and will be described separately.

\subsection{Days $133-139$ of 2004}

The next event presented is from days 133-139 (12-18 May) 2004, when Cassini was at a radial distance of $\sim 400 R_{S}$ from Saturn. The data from this interval are shown in Fig. 2 in the same format as Fig. 1, and summarised in Table 1 under event number 9. The radial propagation delay between Cassini and Saturn is estimated to be $\sim 5 \mathrm{~h}$, varying by $\pm 0.4 \mathrm{~h}$ over the interval due to Cassini's motion toward Saturn. The magnetic field data in the figure clearly show a CIR forward shock at $\sim 18: 00$ UT on day 134 , when the field strength increased from below $0.2 \mathrm{nT}$ to $\sim 0.5 \mathrm{nT}$. The field remained elevated and disturbed for several days, only returning to "quiet" conditions on day 144, when the SKR had already returned to its pre-compression power and periodicity.

The SKR data plotted in the top two panels show seven bursts of SKR during the low field strength interval on days 133-134. The four most powerful bursts, each peaking at $3-5 \times 10^{8} \mathrm{~W} \mathrm{sr}^{-1}$, peaked close to the expected timings marked by the crosses, while the shorter-duration, lower power spikes occurred in addition to the expected times, e.g. $\sim$ 10:00 UT on day 134. At 21:00 UT on day 134 an intense SKR burst was detected, reaching a peak of $\sim 10^{9} \mathrm{~W} \mathrm{sr}^{-1}$. This was $\sim 4 \mathrm{~h}$ after the solar wind shock was expected to arrive at Saturn by employing the $\sim 5 \mathrm{~h}$ propagation delay from the spacecraft to the planet (see the first vertical dashed line), and occurred in between the timings of the expected SKR peaks. A few hours after this initial intensification, and centred at the time of an expected SKR peak, two emission peaks were detected with reduced powers of $\sim 2 \times 10^{8} \mathrm{~W} \mathrm{sr}^{-1}$, which extended to low frequencies $(\sim 10 \mathrm{kHz})$. Over the next two days several bursts of SKR 
Table 1. SKR observations relating to thirteen CIR compression events.

\begin{tabular}{|c|c|c|c|c|c|c|c|c|c|}
\hline \multirow{2}{*}{ No } & \multirow{2}{*}{ Days } & \multirow{2}{*}{$\Delta|\boldsymbol{B}| / \mathrm{nT}$} & \multicolumn{3}{|c|}{ Percentage of SKR peaks with affected powers } & \multirow{2}{*}{$\begin{array}{c}\text { Ratio of powers } \\
\text { across compression }\end{array}$} & \multicolumn{2}{|c|}{ Relative timing of bursts $(\%)$} & \multirow{2}{*}{$\begin{array}{l}\text { Initial SKR burst } \\
\text { at "expected" time? }\end{array}$} \\
\hline & & & Increased & Reduced & Similar $^{\mathrm{a}}$ & & Early & Late & \\
\hline 1 & $001-007$ & 1.0 & 50 & 29 & 21 & 1.35 & 50 & 50 & No \\
\hline 2 & $015-022$ & 0.2 & 7 & 86 & 7 & 0.96 & 43 & 57 & Yes \\
\hline 3 & $025-031$ & 0.5 & 100 & 0 & 0 & 5.64 & 45 & 55 & No \\
\hline 4 & $036-042$ & 0.3 & 0 & 100 & 0 & 0.29 & 69 & 31 & Yes \\
\hline 5 & $042-048$ & 0.2 & 17 & 67 & 17 & 2.34 & 33 & 67 & Yes \\
\hline 6 & 049-055 & 0.5 & 42 & 58 & 0 & 1.5 & 25 & 75 & No \\
\hline 7 & $109-115$ & 0.2 & 83 & 0 & 17 & 1.82 & 33 & 67 & Yes \\
\hline 8 & $119-125$ & -0.5 & 57 & 14 & 29 & 0.32 & 79 & 21 & No \\
\hline 9 & $133-139$ & 0.4 & 27 & 64 & 9 & 3.6 & 45 & 55 & No \\
\hline 10 & $159-166$ & 0.4 & 46 & 54 & 0 & 1.94 & 31 & 69 & Yes \\
\hline $11 a$ & $206-212$ & 0.5 & 58 & 33 & 8 & 1.94 & 50 & 50 & Yes \\
\hline $11 b$ & $213-219$ & -1.1 & 69 & 23 & 8 & 0.38 & 31 & 69 & Yes \\
\hline 12 & $230-236$ & 0.6 & 36 & 36 & 27 & 1.66 & 18 & 82 & Yes \\
\hline 13 & $258-264$ & 0.2 & 62 & 23 & 15 & 4.05 & 15 & 85 & No \\
\hline
\end{tabular}

a $\pm 3 \times 10^{7} \mathrm{~W} \mathrm{sr}^{-1}$

were detected but at relatively low powers (between $\sim 2 \times 10^{7}$ and $\sim 10^{8} \mathrm{~W} \mathrm{sr}^{-1}$ ). Between $\sim$ 14:00 UT and $\sim$ 19:00 UT on day 135 the RPWS antennae were not appropriately orientated with respect to Saturn to reliably determine the wave properties. The SKR data including the burst peak identified during this time are therefore excluded from our analysis. Some of the low-power bursts identified on days 135-137 (disregarding the few-hour interval just mentioned) occurred at the expected timings but there were also peaks in between these times e.g. $\sim 08: 00$ UT on day 136. During days 137 to 138 the SKR emission was intensified again, with a particularly powerful burst lasting from $\sim$ 08:00 UT to 14:00 UT on day 137 , with a maximum power of $\sim 6 \times 10^{8} \mathrm{~W} \mathrm{sr}^{-1}$. This burst has no obvious trigger in the IMF data as indicated by the second vertical dashed line. The powers of the SKR bursts then decreased gradually for the rest of the interval to levels of $\sim 10^{8} \mathrm{~W} \mathrm{sr}^{-1}$. These emission bursts again peaked close to the expected timings, but with other lower power peaks in between.

\subsection{Days 206-219 of 2004}

The final interval presented here includes days 206-219 (24 July-6 August) of 2004, after SOI when Cassini was travelling away from Saturn in the dawn sector, at distances of $\sim 115-140 R_{S}$ (see event number 11 in Table 1 , to be discussed below). However, Cassini was travelling close to the dawn meridian so the displacement in the Sun-planet radial direction was very small, and hence the radial propagation delay was only a few minutes. The solar wind dynamic pressure values derived from the available CAPS ion data over this interval are plotted in the bottom panel of Fig. 3a and b. At the start of the interval, shown in Fig. 3a, the IMF magnitude was low and relatively constant at $\sim 0.1-0.2 \mathrm{nT}$. At $\sim 18: 00$ UT on day 207 a CIR compression was encoun- tered by Cassini causing an increase in the measured field strength to $\sim 0.6-0.8 \mathrm{nT}$, marked on Fig. $3 \mathrm{a}$ by the first vertical dashed line. The field continued to be elevated and disturbed for the next six days, and the HCS was encountered several times. The solar wind dynamic pressure plotted in the bottom panel was similarly low at under $0.01 \mathrm{nPa}$ at the start of the interval, increasing to $\sim 0.02 \mathrm{nPa}$ at the time marked by the vertical dashed line (coincident with the increase in IMF magnitude) due to increases in both the plasma density and velocity, then remaining high and peaking in excess of $0.1 \mathrm{nPa}$ over the next six days. At the start of day 214 (see Fig. 3b) there was an abrupt decrease in field strength from $\sim 1.7 \mathrm{nT}$ to $\sim 0.6 \mathrm{nT}$, which we identify as the CIR reverse shock at the trailing boundary of the compression region. At this time the solar wind dynamic pressure also decreased from $\sim 0.1 \mathrm{nPa}$ to $\sim 0.02 \mathrm{nPa}$, associated with a decrease in the density combined with an increase in the flow speed. After the shock the field magnitude remained approximately constant at $\sim 0.5 \mathrm{nT}$ for over 5 days, until the end of the interval shown. Where available the dynamic pressure data were similarly elevated relative to the start of the interval at values of $\sim 0.01-0.05 \mathrm{nPa}$, but lower than the peak compression values measured during days $208-214$. We note that the excellent correspondence shown here between the IMF and solar wind data sets is typical for those dates when the CAPS plasma data were available for comparison.

Examining the SKR emissions plotted in the upper two panels of Fig. 3a and $b$ at the start of the interval, there were seven emission peaks exceeding the $5 \times 10^{7} \mathrm{~W} \mathrm{sr}^{-1}$ limit before the first vertical dashed line, which are grouped into four longer-duration $(\sim 6-8 \mathrm{~h})$ bursts. These bursts peaked at powers of $\sim 1-3 \times 10^{8} \mathrm{~W} \mathrm{sr}^{-1}$ up to $\sim 2.5 \mathrm{~h}$ before their expected timing in each case. Coincident with the detection of the solar wind shock at Cassini at $\sim$ 18:00 UT on day 207, there was a more powerful burst of SKR, in phase with the 


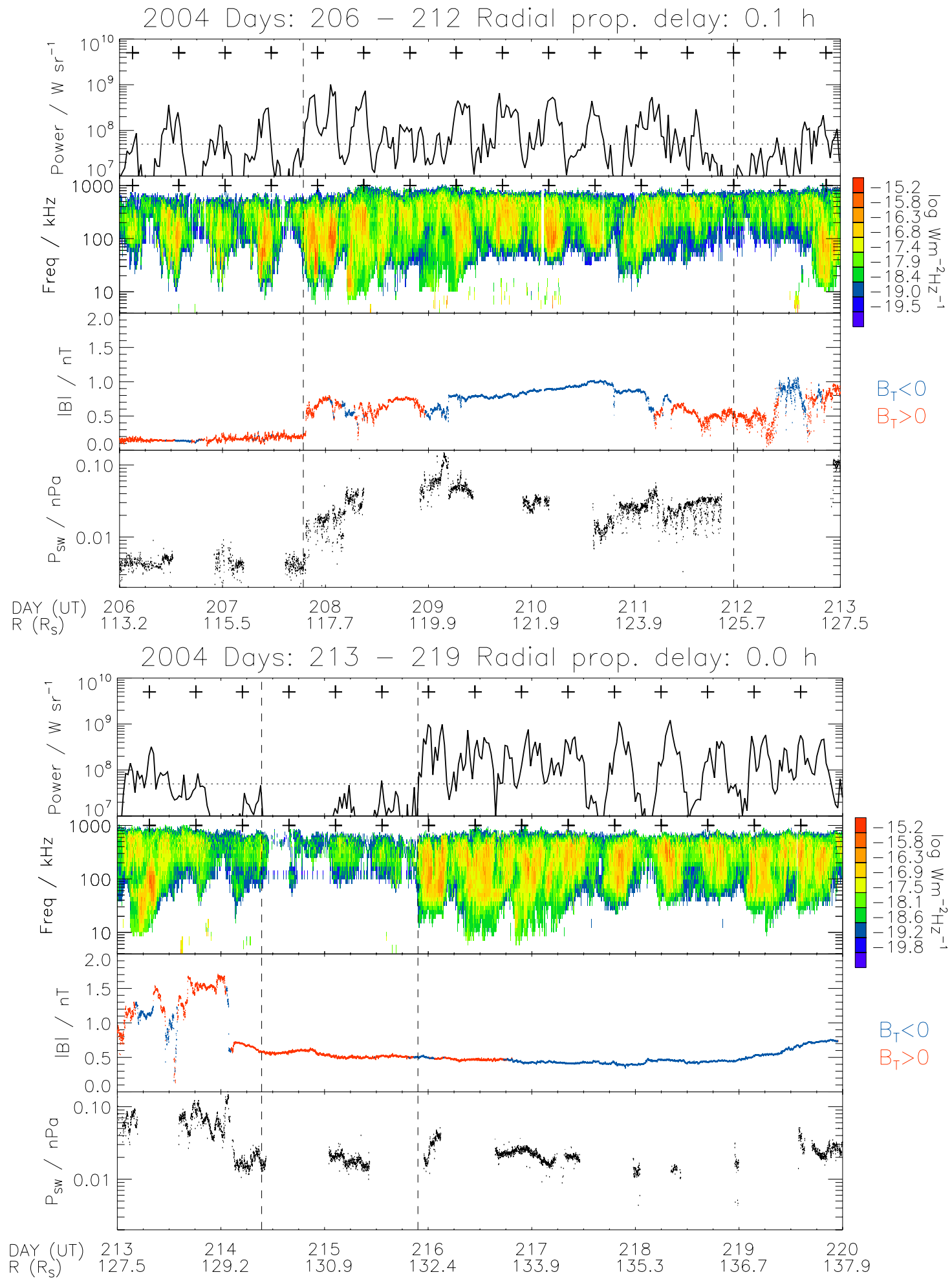

Fig. 3. SKR and IMF data from (a) days 206-212, and (b) days 213-219 of 2004. The data in the top three panels are in the same format as Fig. 1. The SKR power data in the top panel are $0.539056 \mathrm{~h}$ averages. The bottom panel shows the solar wind dynamic pressure in $\mathrm{nPa}$ calculated from CAPS ion measurements. 
preceding bursts, peaking at $\sim 6 \times 10^{8} \mathrm{~W} \mathrm{sr}^{-1}$. This was followed by two more powerful peaks just a few hours apart, then several low power $\left(\sim 10^{8} \mathrm{~W} \mathrm{sr}^{-1}\right)$ bursts over the next two SKR cycles. The second of the intense peaks after the shock arrival was an "extra" peak occurring in between the expected timings of the SKR peaks, and only one of the subsequent low power bursts on day 208 was at the expected timing of a SKR peak ( 20:00 UT on day 208). As in the two previous events, Cassini detected intensified emission around the time of the arrival of the compression, which included an "extra" peak not at the expected timing. In this example though, the successive emissions were less powerful than those preceding the compression. Over days 209212 (i.e. after the two reduced power SKR cycles) a series of long-duration powerful bursts were detected, each with maximum powers of $\sim 5 \times 10^{8} \mathrm{~W} \mathrm{sr}^{-1}$. There were emission peaks close to all expected times but also several extra peaks in between, usually at lower powers. Further low power $\left(<10^{8} \mathrm{~W} \mathrm{sr}^{-1}\right)$ emissions were then detected late on day 211 until mid day 212. Close to midnight on day 211 the SKR power was at a minimum of less than $10^{7} \mathrm{~W} \mathrm{sr}^{-1}$ (marked by the second vertical dashed line), which is a time when an emission peak was expected.

After these low power emissions, two more powerful lower frequency $\left(\sim 2 \times 10^{8} \mathrm{~W} \mathrm{sr}^{-1}, \sim 10-100 \mathrm{kHz}\right)$ bursts were observed at $\sim 20: 00$ UT on day 212 (Fig. 3a) and $\sim 06: 00$ UT on day 213 (Fig. 3b). After $\sim 20: 00$ UT on day 213, just preceding the reverse shock encounter in the solar wind, the SKR emission was at very low power $\left(<5 \times 10^{7} \mathrm{~W} \mathrm{sr}^{-1}\right)$. The dynamic pressure decreased at this time, such that Saturn's magnetosphere would have expanded. Out of the next four SKR peaks expected, only three very low power $\left(<10^{8} \mathrm{~W} \mathrm{sr}^{-1}\right)$ bursts were detected. Powerful SKR emissions reappeared at the end of day 215 coincident with an HCS encounter and increased plasma density, possibly indicating another smaller compression region. These powerful emissions continued, occasionally peaking at powers in excess of $10^{9} \mathrm{~W} \mathrm{sr}^{-1}$, until the end of the interval shown on day 219. There were peaks in emissions close to the expected times, but also many extra peaks of similar powers in between.

\subsection{Summary of all events identified}

Having described the SKR response to three different CIR compression events, these features are now summarised in Table 1 together with the results from all identified events. The three events discussed above are highlighted by italic text (numbers 6, 9 and 11). The first column lists the interval of days surrounding each compression that was studied. Next, the size of the initial solar wind shock $\Delta|\boldsymbol{B}|$ is given for comparison, where a negative value of $\Delta|\boldsymbol{B}|$ implies a reverse shock such as that identified on day 214 (Fig. 3b). The peak powers of the SKR emissions which occurred close to the expected peak times during the five days following the predicted arrival of the shock at Saturn are compared to that of the last "undisturbed" peak, and then categorized as increased, decreased, or similar $\left( \pm 3 \times 10^{7} \mathrm{~W} \mathrm{sr}^{-1}\right)$. Next, the ratio of the peak powers of the two bursts closest to the arrival of the compression (i.e. the last before and the first after) is listed for each event. The subsequent two columns list the proportion of bursts in the few-day post-compression interval that were detected early or late relative to the "expected" Kurth et al. (2007) timings. The maximum time difference allowed was $3 \mathrm{~h}$ either side of the expected time, a value dictated by the quiet-time deviation of the SKR peak timings from the Kurth et al. (2007) fit, and encompassing the short-term fluctuations of the SKR period derived by Zarka et al. (2007). The final column in Table 1 identifies whether the first peak in SKR power following the arrival of the compression occurred close to the time of the next expected peak, or whether it was distinctly out of phase with the Kurth et al. (2007) pulsing.

Considering first the intensity of the SKR pulses there are three additional factors affecting the detection of emission at the spacecraft to take into account. The first of these is the variation of the SKR signal to noise ratio detected by Cassini as its distance from Saturn changed. This can be neglected in the present study because the power level we use to define a burst is considerably greater than the noise level for the entire interval studied. The second influencing factor is that the detected SKR properties can be affected by the configuration of the RPWS antennae (Cecconi and Zarka, 2005), however we have attempted to identify the affected times and disregard the data within. The final and potentially most significant factor for this study is that the SKR detection depends in the direction of emission from the source. This beaming effect is a possible explanation for "missed" bursts of SKR, as will be discussed below.

Referring to the events summarised in Table 1, the peak powers of the initial bursts immediately following the compressions were usually increased by factors of between 1.35 and 5.64, with an average of 2.6. Events 2 and 4 showed reduced powers after the compression because the previous burst was already significantly more powerful than those preceding it. These powerful earlier bursts could in fact be the intense compression response peaks, following the behaviour of the other events, if the propagation delay was overestimated by a few hours in each case. Unfortunately, as explained above, the propagation delay cannot be estimated any more accurately. The initial bursts following the reverse shocks (events 8 and $11 \mathrm{~b}$ - see Fig. 3b for the latter) were reduced by factors of 0.32 and 0.38 respectively. An overall positive correlation was identified between the change in IMF magnitude $\Delta|\boldsymbol{B}|$ and the ratio of the powers across the compression (correlation coefficient $C=0.37$ ). Apart from the initial burst, the SKR peak powers were not affected by solar wind compressions in a consistent manner. The percentage of identified peak powers that were intensified or reduced relative to those before the compression ranged from 
0 to $100 \%$, implying that during one event all the observed bursts were intensified (event 3 ) and during another event all the burst powers were reduced (event 4, explained above). Much more commonly, a combination of intensified and reduced powers was observed. Similar proportions of intensified and reduced bursts were identified overall.

Considering now the timings at which the bursts occurred, we first mention the time delay between the arrival of the compression at Saturn and the time of the next SKR emission peak, which is of interest for understanding the interaction causing the SKR intensification. As already discussed there are large uncertainties in the solar wind propagation delay which make this calculation difficult. Even for the last three events included in this study, when Cassini was relatively close to Saturn such that the estimated propagation delay was an hour or less, there is a large variation in the times calculated: from less than $1 \mathrm{~h}$ to over $10 \mathrm{~h}$ until the start of the burst, with a mean of $\sim 3 \mathrm{~h}$, and from $2 \mathrm{~h}$ to $11 \mathrm{~h}$ (mean value $\sim 4 \mathrm{~h}$ ) until the peak in the emission. The variability of this result inhibits any insight into the SKR generation mechanisms. Looking now at the times the emission peaks were observed relative to the "expected" times, a wide variation in behaviour was identified. During two events (numbers 1 and $11 \mathrm{a}-$ see Fig. 3a for the latter), equal numbers of early and late bursts were identified. In contrast, during event $879 \%$ of the bursts were early, and in event $1385 \%$ were late. As mentioned above the time differences themselves varied from as short as just over one minute, up to nearly as long as the $3 \mathrm{~h}$ maximum set, i.e. a peak in SKR was usually detected within $3 \mathrm{~h}$ of the expected time. A more reliable determination of the changes in SKR pulsing could be made in the future by employing a model of both the long-term (Kurth et al., 2007) and short-term (Zarka et al., 2007) variations in SKR period. Overall, we can deduce, however, that the time differences of the post-compression bursts were not significantly different to those before the compression. In most cases some extra bursts of similar or lower powers were also detected, and these were also seen during the pre-compression intervals e.g. the start of event 6 , shown in Fig. 1. The first intense burst of SKR following the compression was observed as a distinct peak (i.e. more than $3 \mathrm{~h}$ before the next expected SKR peak) in five of the events identified, e.g. events 6 and 9 shown in Figs. 1 and 2.

\section{Discussion and conclusions}

It is evident from the descriptions above that a variety of features in the SKR data are detected following a solar wind disturbance. We now discuss common features from all the events included in this study, and suggest possible explanations for these observations. One recurrent feature in the SKR data is the initial high-power burst, usually peaking at $\sim 10^{9} \mathrm{~W} \mathrm{sr}^{-1}$, which occurred around the time of the arrival of the solar wind compression at the magnetosphere. (The exact timing is approximate due to uncertainties in the propagation delay.) The timing of this burst is sometimes out of phase with the expected times of the SKR emission peaks to such an extent that it cannot be attributed to the intrinsic deviation of the bursts around the Kurth et al. (2007) fit. The opposite effect, i.e. a decrease in SKR emitted power, is observed after the two reverse shocks identified in the data. After the initial SKR burst, the behaviour of the SKR varied between events. In four of the events identified, the SKR pulses continued at high powers for several days (e.g. days 49-55, shown in Fig. 1). Conversely, during event 3 (25-31 January 2004, not shown here), very little SKR emission was detected by Cassini for $\sim 18 \mathrm{~h}$ shortly after the disturbance (Jackman et al., 2005). Also, very low levels of emission were detected for $\sim 30 \mathrm{~h}$ following the reverse shock on day 214 shown in Fig. 3b. Most commonly in the events studied, periods of both intense and weak SKR bursts (relative to those preceding the solar wind disturbance) were detected after solar wind compressions (e.g. days 133-139 shown in Fig. 2). In general, this study confirms that the emitted SKR power initially increased when the solar wind was compressed. In addition, this study presents two examples of a reduction in emitted SKR power when a reverse shock in the solar wind was encountered. After this initial post-compression or reverse shock burst, the behaviour is variable: sometimes intensified and sometimes reduced, with no obvious cause in the IMF data.

Now considering the modulation of the SKR bursts, we note that overall the SKR continues to pulse close to the expected times (with respect to the Kurth et al. (2007) drifting period) during and after the solar wind disturbances, when examined over timescales significantly longer than the few minute variations noted by Zarka et al. (2007), and allowing for a similar deviation in time to that observed during the non-compression intervals. There are two exceptions to this. First, the initial powerful SKR burst described above does not always fall coincident with a regular pulse of the SKR e.g. on day 51 shown in Fig. 1. Also, there are the two occasions mentioned above where the SKR emission detected becomes very low. In these cases at least one SKR "beat" is skipped, but when the SKR pulses return, they are in phase with the Kurth et al. (2007) pulsing (see Fig. 3b). In addition to the regular pulsing of the SKR, there are numerous "extra" bursts at the same frequencies, of lesser or equal power, e.g. days 208-212 in Fig. 3a.

Since there is as yet no complete theory on how SKR is generated and controlled we do not attempt to give detailed physical reasons for the observed variations in the emitted power and phasing of the SKR. We can, however, suggest how our observations may relate to those presented in other studies. For example, Kurth et al. (2005a) noted that intense SKR bursts were coincident with enhanced UV auroral emissions during the January 2004 HST imaging campaign. Cowley et al. (2005) attributed these enhanced auroral emissions to large reconnection events in the magnetotail, 
which enhance the associated field-aligned currents. Such enhanced currents are then a possible explanation for the intense bursts of SKR observed shortly after the arrival of a solar wind compression at Saturn's magnetosphere. Similarly, any intense "extra" peaks of SKR over the next few days could be associated with further reconnection events, as described by Bunce et al. (2005), Jackman et al. (2005) and Mitchell et al. (2005). This is analogous to the behaviour of terrestrial auroral kilometric radiation, which is enhanced during reconnection events in the Earth's magnetotail (e.g. Gurnett, 1974). The frequent detection of the "extra" bursts also affirms that parameters other than planetary rotation and the solar wind conditions could affect the occurrence of SKR bursts, e.g. the location of Saturn's moons (Menietti et al., 2007) which appear to influence the occurrence of tail reconnection events (Russell et al., 2008).

Kurth et al. (2005a) discussed in depth event 3 mentioned above (25-31 January 2004, not shown here), when the SKR detections became very weak, and stated that although Cassini RPWS detected very little SKR emission, the Unified Radio and Plasma wave experiment onboard the Ulysses spacecraft did measure SKR at the expected time. They therefore attributed the lack of measurements by Cassini to the source beaming effect mentioned above. Once the source beaming phenomenon is satisfactorily understood and modelled, its effects can be systematically accounted for when studying SKR data series such as those included in this study.

To sum up our discussion of the SKR phasing, our analysis suggests that while the period of the SKR pulses is varying slowly over time (e.g. Galopeau and Lecacheux, 2000; Gurnett et al., 2005; Kurth et al., 2007), solar wind compressions do not have a significant effect on the pulsing of the SKR over the few-day timescales considered here. In the vast majority of our observations there continue to be SKR emission peaks at the expected times, as well as additional bursts detected under compression and rarefaction conditions. While SKR bursts during the compression event are intensified or reduced relative to those before the event, they usually continue to pulse at the same period as the preceding ones. The pulsing of the bursts is therefore the same before and after the compression events.

In conclusion, we have presented thirteen examples of Cassini measurements of compressions in the solar wind, and the corresponding SKR emissions observed by the spacecraft. We have examined the relative intensities of the SKR bursts before, during, and after the solar wind disturbances, and also compared the timings of the observed pulses with those predicted using the Kurth et al. (2007) drifting SKR period. The behaviour of the SKR following the compressions was variable, however, we are able to draw some general conclusions from analysis of the thirteen events included in this study. Firstly we confirm that there was an overall positive correlation between the change in solar wind dynamic pressure (using $\Delta|\boldsymbol{B}|$ as a proxy) and the change in emitted SKR power, as identified in Voyager data (Desch,
1982; Desch and Rucker, 1983), with a correlation coefficient $C=0.37$. The timings of the initial SKR intensifications following the compressions can be independent of the longterm phasing of the SKR bursts, but during the disturbed interval the SKR continues to pulse close to the expected times, within the tens of minutes variations observed before the compressions. Distinct "extra" bursts of SKR emission were also detected both before and during the compressions. The intensity of the detected emissions during the disturbed intervals is variable, sometimes remaining intense for several days, sometimes reducing, and rarely disappearing. This result warrants further investigation in relation to theories on the origin of SKR, and demonstrates that the SKR emissions cannot be simply used as a diagnostic of the prevalent solar wind conditions (e.g. when Cassini is inside Saturn's magnetosphere) as was suggested before Cassini SOI (e.g. Zarka and Kurth, 2005), without careful consideration of other influencing factors. This is in agreement with recent conclusions drawn by Rucker et al. (2008) following calculation of solar wind-SKR correlation coefficients. Our final conclusion is that, although variations in the SKR intensity are observed, in general the modulation of the SKR bursts over these few-day timescales was consistent before and after the solar wind CIR compressions.

Acknowledgements. We thank John Steinberg and the LANL CAPS team for providing the Cassini ion data from which the solar wind dynamic pressure values were calculated. We thank Chris Arridge (UCL/MSSL) for providing a description of the relevant CAPS ELS data (Lead Co-I: Andrew Coates UCL/MSSL). Work at Leicester was supported by STFC grant PP/E000983/1. SVB was also funded by an STFC studentship and SWHC by a Royal Society Leverhulme Trust Senior Research Fellowship.

Topical Editor I. A. Daglis thanks two anonymous referees for their help in evaluating this paper.

\section{References}

Bunce, E. J., Cowley, S. W. H., Wright, D. M., Coates, A. J., Dougherty, M. K., Krupp, N., Kurth, W. S., and Rymer, A. M.: In-situ observations of a solar wind compression-induced hot plasma injection in Saturn's tail, Geophys. Res. Lett., 32, L20S04, doi:10.1029/2005GL022888, 2005.

Cecconi, B. and Zarka, P.: Direction finding and antenna calibration through analytical inversion of radio measurements performed using a system of two or three electric dipole antennas on a three-axis stabilized spacecraft, Radio Sci., 40, RS3003, doi:10.1029/2004RS003070, 2005.

Cowley, S. W. H., Badman, S. V., Bunce, E. J., Clarke, J. T., Gérard, J.-C., Grodent, D., Jackman, C. M., Milan, S. E., and Yeoman, T. K.: Reconnection in a rotation-dominated magnetosphere and its relation to Saturn's auroral dynamics, J. Geophys. Res., 110, A02201, doi:10.1029/2004JA010796, 2005.

Crary, F. J., Clarke, J. T., Dougherty, M. K., Hanlon, P. G., Hansen, K. C., Steinberg, J. T., Barraclough, B. L., Coates, A. J., Gérard, J.-C., Grodent, D., Kurth, W. S., Mitchell, D. G., Rymer, A. M., and Young, D. T.: Solar wind dynamic pressure 
and electric field as the main factors controlling Saturn's auroras, Nature, 433, 720, doi:10.1038/nature03333, 2005.

Desch, M. D.: Evidence for solar wind control of Saturn radio emission, J. Geophys. Res., 87, 4549-4554, 1982.

Desch, M. D. and Kaiser, M. L.: Voyager measurement of the rotation period of Saturn's magnetic field, Geophys. Res. Lett., 8, 253-256, 1981.

Desch, M. D. and Rucker, H. O.: The relationship between Saturn kilometric radiation and the solar wind, J. Geophys. Res., 88, 8999-9006, 1983.

Dougherty, M. K., Kellock, S., Southwood, D. J., Balogh, A., Smith, E. J., Tsurutani, B. T., Gerlach, B., Glassmeier, K.-H., Gleim, F., Russell, C. T., Erdos, G., Neubauer, F. M., and Cowley, S. W. H.: The Cassini magnetic field investigation, Space Sci. Rev., 114, 331-383, 2004.

Galopeau, P. H. M. and Lecacheux, A.: Variations of Saturn's radio rotation period at kilometre wavelengths, J. Geophys. Res., 105, 13 089-13 102, 2000.

Gosling, J. T. and Pizzo, V. J.: Formation and evolution of corotating interaction regions and their three dimensional structure, Space Sci. Rev., 89, 21-52, 1999.

Gurnett, D. A.: The earth as a radio source: terrestrial kilometric radiation, J. Geophys. Res., 79, 4227-4238, 1974.

Gurnett, D. A., Kurth, W. S., Kirchner, D. L., Hospodarsky, G. B., Averkamp, T. F., Zarka, P., Lecacheux, A., Manning, R., Roux, A., Canu, P., Cornilleau-Wehrlin, N., Galopeau, P., Meyer, A., Boström, R., Gustafsson, G., Wahlund, J.-E., Åhlen, L., Rucker, H. O., Ladreiter, H. P., Macher, W., Woolliscroft, L. J. C., Alleyne, H., Kaiser, M. L., Desch, M. D., Farrell, W. M., Harvey, C. C., Louarn, P., Kellogg, P. J., Goetz, K., and Pedersen, A.: The Cassini Radio and Plasma Wave Investigation, Space Sci. Rev., 114, 395-463, 2004.

Gurnett, D. A., Kurth, W. S., Hospodarsky, G. B., Persoon, A., Averkamp, T. F., Cecconi, B., Lecacheux, A., Zarka, P., Canu, P., Cornilleau-Wehrlin, N., Galopeau, P., Roux, A., Harvey, C., Louarn, P., Boström, R., Gustafsson, G., Wahlund, J.-E., Desch, M. D., Farrell, W. M., Kaiser, M. L., Goetz, K., Kellogg, P. J., Fischer, G., Ladreiter, H.-P., Rucker, H. O., Alleyne, H., and Pedersen, A.: Radio and plasma wave observations at Saturn from Cassini's approach and first orbit, Science, 307, 12551259, 2005.

Jackman, C. M., Achilleos, N., Bunce, E. J., Cecconi, B., Clarke, J. T., Cowley, S. W. H., Kurth, W. S., and Zarka, P.: Interplanetary conditions and magnetospheric dynamics during the Cassini orbit insertion fly-through of Saturn's magnetosphere, J. Geophys. Res., 110, A10212, doi:10.1029/2005JA011054, 2005.

Kaiser, M. L., Desch, M. D., Warwick, J. W., and Pearce, J. B.: Voyager detection of non-thermal radio emission from Saturn, Science, 209, 1238-1240, 1980.
Kurth, W. S., Gurnett, D. A., Clarke, J. T., Zarka, P., Desch, M. D., Kaiser, M. L., Cecconi, B., Lecacheux, A., Farrell, W. M., Galopeau, P., Gérard, J.-C., Grodent, D., Prangé, R., Dougherty, M. K., and Crary, F. J.: An Earth-like correspondence between Saturn's auroral features and radio emission, Nature, 433, 722-725, 2005.

Kurth, W. S., Gurnett, D. A., Cecconi, B., Zarka, P., Lecacheux, A., Kaiser, M. L., Desch, M. D., Farrell, W. M., Galopeau, P., Louarn, P., and Rucker, H. O.: Saturn's radio emissions, Magnetospheres of the Outer Planets 2005 Programme and Abstracts, p. 76, University of Leicester, Leicester, UK, 2005.

Kurth, W. S., Lecacheux, A., Averkamp, T. F., Groene, J. B., and Gurnett, D. A.: A Saturnian longitude system based on a variable kilometric radiation period, Geophys. Res. Lett., 34, L02201, doi:10.1029/2006GL028336, 2007.

Lamy, L., Zarka, P., Cecconi, B., Prangé, R., Kurth, W. S., and Gurnett, D. A.: Saturn Kilometric Radiation: average and statistical properties, J. Geophys Res., 113, A07201, doi:10.1029/2007JA012900, 2008.

Menietti, J. D., Groene, J. B., Averkamp, T. F., Hospodarsky, G. B., Kurth, W. S., Gurnett, D. A., and Zarka, P.: Influence of Saturnian moons on Saturn kilometric radiation, J. Geophys. Res., 112, A09211, doi:10.1029/2007JA012331, 2007.

Mitchell, D. G., Brandt, P. C., Roelof, E. C., Dandouras, J., Krimigis, S. M., Mauk, B. H., Paranicas, C. P., Krupp, N., Hamilton, D. C., Kurth, W. S., Zarka, P., Dougherty, M. K., Bunce, E. J., and Shemansky, D. E.: Energetic ion acceleration in Saturn's magnetotail: Substorms on Saturn?, Geophys. Res. Lett., 32(20), L20S01, doi:10.1029/2005GL022647, 2005.

Rucker, H. O., Panchenko, M., Hansen, K. C., Taubenschuss, U., Boudjada, M. Y., Kurth, W. S., Dougherty, M. K., Steinberg, J. T., Zarka, P., Galopeau, P. H. M., McComas, D. J., and Barrow, C. H.: Saturn Kilometric Radiation as a monitor for the solar wind?, Adv. Space. Res., 42, 40-47, doi:10.1016/j.asr.2008.02.008, 2008.

Russell, C. T., Jackman, C. M., Wei, H. Y., Bertucci, C., and Dougherty, M. K.: Titan's influence on Saturnian substorm occurrence, Geophys. Res. Lett., 35, L12105, doi:10.1029/2008GL034080, 2008.

Smith, E. J. and Wolfe, J. H.: Observations of interaction regions and corotating shocks between one and five AU: Pioneers 10 and 11, Geophys. Res. Lett., 3(3), 137-140, 1976.

Wu, C. S. and Lee, L. C.: A theory of the terrestrial kilometric radiation, Astrophys. J., 230, 621-626, 1979.

Young, D. T., Berthelier, J. J., Blanc, M., et al.: Cassini Plasma Spectrometer Investigation, Space Sci. Rev., 114, 1-112, 2004.

Zarka, P. and Kurth, W. S.: Radio wave emission from the outer planets before Cassini, Space Sci. Rev., 116, 37-397, 2005.

Zarka, P., Lamy, L., Cecconi, B., Prangé, R., and Rucker, H. O.: Modulation of Saturn's radio clock by solar wind speed, Nature, 450, 265-267, 2007. 